\title{
CAUCHY BVP FOR ELASTIC HALF-PLANE POSED IN DISPLACEMENT ORIENTATIONS
}

\author{
ALEXANDER N. GALYBIN \\ The Schmidt Institute of Physics of the Earth (IPE RAS), Russia
}

\begin{abstract}
This study presents a Cauchy-type boundary value problem of plane elasticity in which the boundary conditions are posed in terms of the orientations of the displacement vector and its normal derivative. No magnitudes of the displacements are specified. The problem is reduced to a singular integral equation by using the well-known Muskhelishvili's theory based on the complex potentials. The solvability of the integral equation is analysed in accordance with the Gakhov's approach, which reveals that the problem has a finite number of linearly independent solutions depending on the index of the corresponding Riemann BVP. The index is defined through the orientations of the contour displacements. More detailed analysis is performed for the case of elastic half-plane since previously it has been shown that the shape of the domain does not influence the solvability. A numerical approach for solving the problem for the arbitrary domain is outlined.
\end{abstract}

Keywords: plane elasticity, boundary value problems, complex potentials, singular integral equations.

\section{INTRODUCTION}

This study continues the investigation of the incorrectly posed boundary value problems, BVP, of plane elasticity which formulations employ the boundary magnitudes of neither stresses nor displacements. Such formulations are found in geomechanics where the magnitudes of forces/displacements acting on the margins of tectonic plates forming the earth's crust are unknown. Instead the orientations of principal stresses and displacements are known not only near the margins but inside the plates as well. This type of data has become the main motivation for the development of non-standard models for the stress analysis by formulation of incorrectly posed BVPs [1]-[6]. The main feature of these problems is non-uniqueness of the solution, which is expressed as the presence of certain number of arbitrary parameters entered into the general solution for the stress (displacement) field. These, in principle, can be identified if the magnitudes of stresses (displacements) are measured at a number of discrete points.

This investigation is mainly based on the following approach used previously. Firstly, the boundary conditions in terms of the stress/displacement orientations are formulated through the boundary values of the complex potentials (holomorphic functions in the domain considered). This allows one to reduce the BVP to a system of singular integral equations, SIE, for the determination of unknown auxiliary functions that are usually proportional to the jump of the displacements across the contour (provided that the stress vector is continuous across the contour). The obtained system of SIE can be converted into a single complex SIE that contains the unknown complex valued function, its complex conjugate, the singular and regular integrals and their conjugates. Gakhov [7] has suggested the following approach suggested for analysing the solvability of the complex SIE. The first step assumes separation of the complex SIE in two parts. The first (dominant) part contains non-integral and singular terms while the second part contains all regular terms. The solvability of the full SIE is defined by its dominant part only. Therefore, as the second step, the dominant SIE is reduced to the Riemann BVP by applying a special integral operator specified by the coefficients of the dominant SIE (see Section 3 for more details). 
Finally, the solvability of the Riemann BVP depends on the index determined by the coefficient of the Riemann problem. The latter is calculated as the increment of its argument (divided by $2 \pi$ ) after traversing the contour anticlockwise. If the index is positive then the Riemann problem has a finite number of solutions that is proportional to the index, otherwise the BVP does not have solutions in the class of holomorphic functions. This approach can also be adopted for numerical solving complete complex SIEs [6].

It is shown in [1]-[6] that the dominant SIE corresponds to the case of elastic half-plane regardless of the boundary condition used. Therefore we analyse the dominant SIE only, i.e. the case of half-plane, which makes it easy to operate with mathematical transformations but still keeps the necessary generality. In the case considered further on we arrive at one single complex SIE instead of a system of two real SIEs as e.g. in [1] or [5], [6] that can be solved successively. Therefore one modification of the previously reported technique is proposed to reduce the problem to the SIE suitable for Gakhov's approach. We also outline a numerical approach that is not limited to the dominant SIE but is applicable for the full SIE as well.

\section{PROBLEM FORMULATION AND INTEGRAL EQUATIONS}

\subsection{Preliminaries}

Let us introduce the following notations for the complex displacement vector

$$
W \equiv 2 G\left(u_{1}+i u_{2}\right)=\omega \exp (i \beta),
$$

and refer to the Kolosov-Muskhelishvili formula [8] for the displacements

$$
W=\kappa \phi(z)-z \overline{\Phi(z)}-\overline{\psi(z)} .
$$

Here $G$ is the shear modulus, $u_{i}(i=1,2)$ are the displacement components, $\omega \geq 0$ and $\beta$ are respectively the modulus and the argument of the complex-valued function $W=W(z, \bar{z})$, where $z=x_{1}+i x_{2}, \quad \bar{z}=x_{1}-i x_{2}$. For any domain $\Omega$ bounded by the contour $\Gamma$ the complex potentials in (2) can be expressed through two auxiliary functions $g(t)$ and $h(t)$ as follows

$$
\phi(z)=\frac{1}{2 \pi i} \int_{\Gamma} \frac{g(t) d t}{t-z}, \Phi(z)=\phi^{\prime}(z)=\frac{1}{2 \pi i} \int_{\Gamma} \frac{g^{\prime}(t) d t}{t-z}, \psi(z)=\frac{1}{2 \pi i} \int_{\Gamma} \frac{h(t) d t}{t-z}, z \in \Omega, z \bar{\in} \Gamma .
$$

The boundary values of the displacement vector can be found by the Sokhotski-Plemelj formulas in the form

$$
2 W(\zeta)= \pm\left(\kappa g(\zeta)-\zeta \overline{g^{\prime}(\zeta)}-\overline{h(\zeta)}\right)+\kappa \mathbf{S}(g)-\zeta \overline{\mathbf{S}\left(g^{\prime}\right)}-\overline{\mathbf{S}(h)},
$$

where $+/$ - correspond to the interior/exterior domain respectively and the following notation for the singular integral is introduced

$$
\mathbf{S}(g)=\frac{1}{\pi i} \int_{\Gamma} \frac{g(t)}{t-\zeta} d t, \quad \zeta \in \Gamma .
$$

The functions $g(t)$ and $h(t)$ are not independent. Let us further select

$$
h(\zeta)=\kappa \overline{g(\zeta)}-\bar{\zeta} g^{\prime}(\zeta)
$$


which provides the continuity of the displacements across the contour. These can be presented as follows

$$
W(\zeta)=\kappa \mathbf{S}(g)-\frac{1}{2} \overline{\mathbf{S}\left((\bar{\zeta}-\bar{t}) g^{\prime}\right)}
$$

It should be noted that the second term in the right hand side of (7) is not singular because

$$
\lim _{t \rightarrow \zeta} \frac{\bar{t}-\bar{\zeta}}{t-\zeta}=\frac{d \bar{\zeta}}{d \zeta}=e^{-2 i \theta(\zeta)},
$$

where $\theta$ is the angle between the tangent to the contour and the real axis.

\subsection{Boundary conditions}

Let us assume that the orientation of the displacement vector, angle $\beta=\beta(\zeta)$, is known together with its normal derivative $\beta_{n}^{\prime}(\zeta)$. The first condition means that $\arg W(\zeta)=\beta(\zeta)$ and leads to the boundary condition of the form

$$
\operatorname{Im}\left(e^{-i \beta(\zeta)} W(\zeta)\right)=0 \Leftrightarrow e^{-i \beta(\zeta)} W(\zeta)-e^{i \beta(\zeta)} \overline{W(\zeta)}, \quad \zeta \in \Gamma .
$$

This equation expresses the fact that $e^{-i \beta} W=\omega$ is real everywhere in the domain including its boundary. By differentiation of $\operatorname{Im}\left(e^{-i \beta} W\right)=0$ with respect to outward normal $\boldsymbol{n}$ one finds

$$
e^{-i \beta(\zeta)} W_{n}^{\prime}(\zeta)-e^{i \beta(\zeta)} \overline{W_{n}^{\prime}(\zeta)}=i \beta_{n}^{\prime}(\zeta)\left(e^{-i \beta(\zeta)} W(\zeta)+e^{i \beta(\zeta)} \overline{W(\zeta)}\right), \quad \zeta \in \Gamma .
$$

Taking (9) into account one can rewrite the right-hand side of (10) and present the second boundary condition in the form

$$
e^{-i \beta(\zeta)} W_{n}^{\prime}(\zeta)-e^{i \beta(\zeta)} \overline{W_{n}^{\prime}(\zeta)}=2 i \beta_{n}^{\prime}(\zeta) e^{-i \beta(\zeta)} W(\zeta), \quad \zeta \in \Gamma .
$$

The derivatives of the complex-valued function $f=f(\zeta)$ on the contour $\Gamma$ are defined as their limiting values. Respectively the tangent and normal derivatives are presented as follows

$$
\begin{gathered}
\frac{\partial f}{\partial \boldsymbol{l}}=\boldsymbol{l} \lim _{z \rightarrow \zeta} \frac{\partial f}{\partial z}+\overline{\boldsymbol{l}} \lim _{z \rightarrow \zeta} \frac{\partial f}{\partial \bar{z}}=\zeta^{\prime} \lim _{z \rightarrow \zeta} \frac{\partial f}{\partial z}+\overline{\zeta^{\prime}} \lim _{z \rightarrow \zeta} \frac{\partial f}{\partial \bar{z}}, \\
\frac{\partial f}{\partial \boldsymbol{n}}=\boldsymbol{n} \lim _{z \rightarrow \zeta} \frac{\partial f}{\partial z}+\overline{\boldsymbol{n}} \lim _{z \rightarrow \zeta} \frac{\partial f}{\partial \bar{z}}=-i \zeta^{\prime} \lim _{z \rightarrow \zeta} \frac{\partial f}{\partial z}+i \bar{\zeta}^{\prime} \lim _{z \rightarrow \zeta} \frac{\partial f}{\partial \bar{z}} .
\end{gathered}
$$

Here one should notice that the derivatives in the left hand side are taken with respect to the arc length $s$, i.e. they can be expressed through the derivatives with respect to the complex variable $\zeta$ on the contour by the following relationship

$$
\frac{\partial f}{\partial \boldsymbol{l}}=\frac{\partial f}{\partial s}=\frac{\partial f}{\partial \zeta} \zeta^{\prime}=e^{i \theta} \frac{\partial f}{\partial \zeta} .
$$

By taking into account that $\zeta^{\prime}=\exp (i \theta)$ one present the derivatives of the vector $W$ on the contour $\Gamma$ in the form 


$$
\begin{gathered}
W_{l}^{\prime}(\zeta)=e^{i \theta(\zeta)}(\kappa \Phi(\zeta)-\overline{\Phi(\zeta)})-e^{-i \theta(\zeta)}\left(\zeta \overline{\Phi^{\prime}(\zeta)}+\overline{\Psi(\zeta)}\right), \\
W_{n}^{\prime}(\zeta)=-i e^{i \theta(\zeta)}(\kappa \Phi(\zeta)-\overline{\Phi(\zeta)})-i e^{-i \theta(\zeta)}\left(\zeta \overline{\Phi^{\prime}(\zeta)}+\overline{\Psi(\zeta)}\right),
\end{gathered}
$$

where

$$
\Psi(z)=\psi^{\prime}(z)=\frac{1}{2 \pi i} \int_{\Gamma} \frac{\kappa e^{-2 i \theta(t)} \overline{g^{\prime}(t)}-e^{-2 i \theta(t)} g^{\prime}(t)-\overline{\operatorname{tg}}^{\prime \prime}(t) d t}{t-z}, z \in \Omega, z \bar{\epsilon} \Gamma .
$$

It is evident from eqns (12), (13) that

$$
\begin{gathered}
W_{l}^{\prime}(\zeta)+i W_{n}^{\prime}(\zeta)=2 e^{i \theta(\zeta)}(\kappa \Phi(\zeta)-\overline{\Phi(\zeta)}), \\
W_{l}^{\prime}(\zeta)-i W_{n}^{\prime}(\zeta)=-2 e^{-i \theta(\zeta)}\left(\zeta \overline{\Phi^{\prime}(\zeta)}+\overline{\Psi(\zeta)}\right) .
\end{gathered}
$$

The latter formulas can be rewritten by using complex conjugation of (19) and by passing to the complex derivative using (14) that results in the following expressions

$$
\begin{gathered}
W_{z}^{\prime}(\zeta)=\kappa \Phi(\zeta)-\overline{\Phi(\zeta)}, \\
\bar{W}_{z}^{\prime}(\zeta)=-\bar{\zeta} \Phi^{\prime}(\zeta)-\Psi(\zeta) .
\end{gathered}
$$

Note that these can be directly obtained by direct differentiation of (2). Eqns (18)-(21) provide the link between the tangential and normal derivatives and the boundary values of the derivatives with respect to $z$ and its conjugate on the contour $\Gamma$. It is evident that if the boundary value of any complex-valued function is known than the tangential derivative is also known and if its normal derivative is known then one can find the boundary values of the derivatives with respect to $z$.

Let us combine two real conditions (9) and (11) into one complex by differentiation of $\operatorname{Im}\left(e^{-i \beta} W\right)=\operatorname{Im}(\omega)=0$ with respect to the variable $z$. Similarly to (11) one finds

$$
e^{-i \beta(\zeta)} W_{z}^{\prime}(\zeta)-e^{i \beta(\zeta)} \bar{W}_{z}^{\prime}(\zeta)=2 i \beta_{z}^{\prime}(\zeta) e^{-i \beta(\zeta)} W(\zeta), \quad \zeta \in \Gamma .
$$

However in contrast to (11) this form is now complex and combines two real conditions equivalent to (9) and (11), therefore it can be used for obtaining a complex SIE.

\subsection{Complex singular integral equation}

Let us substitute (2) into the right hand side of (22) and (20) and (21) into the left hand side of (22) to obtain the following boundary value problem for the determination of the holomorphic functions $\varphi, \psi$ and their derivatives

$$
e^{-i \beta(\zeta)}(\kappa \Phi(\zeta)-\overline{\Phi(\zeta)})+e^{i \beta(\zeta)}\left(\bar{\zeta} \Phi^{\prime}(\zeta)+\Psi(\zeta)\right)=2 i \beta_{z}^{\prime}(\zeta) e^{-i \beta(\zeta)}(\kappa \phi(\zeta)-z \overline{\Phi(\zeta)}-\overline{\psi(\zeta)}), \quad \zeta \in \Gamma .(23
$$

Now it is possible to derive a SIE by substituting the boundary values of the complex potentials into (23) followed by the application of the Sokhotski-Plemelj formulas. However this would lead to a SIE that contains both unknown function $g(\zeta)$ and its derivative. No direct techniques for investigation of such SIEs are known, which necessitates further transformation in order to obtain a SIE of the Gakhov's type mentioned above. 
Bearing in mind the relationship between the displacement vector and the unknown density of the Cauchy-type integral one can substitute (7) into (9) to obtain the following relationship

$$
0=e^{-i \beta(\zeta)} W(\zeta)-e^{i \beta(\zeta)} \overline{W(\zeta)}=e^{-i \beta(\zeta)} \kappa \mathbf{S}(g)-e^{i \beta(\zeta)} \kappa \overline{\mathbf{S}(g)}-i \operatorname{Im}\left[e^{i \beta(\zeta)} \mathbf{S}\left((\bar{\zeta}-\bar{t}) g^{\prime}\right)\right], \quad \zeta \in \Gamma .
$$

Here the last term in the right-hand side is not singular.

By differentiation of (24) with respect to $\zeta$ one finds

$$
\begin{aligned}
& 2 i \beta_{\zeta}^{\prime}(\zeta)\left(e^{-i \beta(\zeta)} \kappa \mathbf{S}(g)+e^{i \beta(\zeta)} \bar{\kappa} \mathbf{S}(g)\right)= \\
& =e^{-i \beta(\zeta)} \kappa \mathbf{S}\left(g^{\prime}\right)-e^{i \beta(\zeta)} \kappa \overline{\mathbf{S}\left(e^{-2 i \theta(\zeta)} g^{\prime}\right)}-i \frac{d}{d \zeta} \operatorname{Im}\left[e^{i \beta(\zeta)} \mathbf{S}\left((\bar{\zeta}-\bar{t}) g^{\prime}\right)\right], \quad \zeta \in \Gamma .
\end{aligned}
$$

This provides the relationship between the unknown function and its contour derivative that is further considered as the only unknown function after transformation of the right-hand side of (22) and (23). The left hand sides of (23) can be also transformed in view of the Sokhotski-Plemelj formulas, which eventually leads to the following complex SIE

$$
\begin{aligned}
& e^{-i \beta}\left[ \pm\left(\kappa g^{\prime}-\bar{g}^{\prime}\right)+\mathbf{S}\left(g^{\prime}\right)-\overline{\mathbf{S}\left(g^{\prime}\right)}\right]+e^{i \beta}\left[ \pm e^{2 i \theta}\left(\kappa g^{\prime}-\bar{g}^{\prime}\right)+\overline{\mathbf{S}\left(e^{-2 i \theta}\left(\kappa g^{\prime}-\bar{g}^{\prime}\right)\right)}+\frac{d}{d \zeta} \mathbf{S}\left((\bar{\zeta}-\bar{t}) g^{\prime}\right)\right] \\
& \left.-\kappa \frac{\beta_{z}^{\prime}}{\beta_{\zeta}^{\prime}}\left(e^{-i \beta} \mathbf{S}\left(g^{\prime}\right)-e^{i \beta} \overline{\mathbf{S}\left(e^{-2 i \theta} g^{\prime}\right)}-\frac{i}{\kappa} \frac{d}{d \zeta} \operatorname{Im}\left[e^{i \beta} \mathbf{S}(\bar{\zeta}-\bar{t}) g^{\prime}\right)\right]\right)=0, \quad \zeta \in \Gamma .
\end{aligned}
$$

Eqn (26) represents the full complex SIE for the problem considered. It should be complemented by the integral conditions expressing the fact that the function $g$ returns to its initial value after the complete traverse of the contour,

$$
\int_{\Gamma} g^{\prime}(t) d t=0
$$

\section{THE CASE OF HALF-PLANE}

\subsection{The SIE for half-plane}

It has been mentioned above that solvability of the BVP can be investigated for the case of half-plane, for which the formulas of the previous section can be readily simplified by assuming $\theta=0, \operatorname{Im} \zeta=0$, in particular the singular operator assumes the following form

$$
\mathbf{S}(g)=\frac{1}{\pi i} \int_{-\infty}^{\infty} \frac{g(t)}{t-\zeta} d t, \quad \operatorname{Im} \zeta=0 .
$$

It obeys the following properties

$$
\begin{aligned}
& \mathbf{S}[\mathbf{S}(g)]=g, \\
& \overline{\mathbf{S}(g)}=-\mathbf{S}(\bar{g}) .
\end{aligned}
$$

The former is valid for any closed contour, while the latter is valid only for the real axis.

It is also evident that due to (27) 


$$
\mathbf{S}\left((\bar{\zeta}-\bar{t}) g^{\prime}\right)=0
$$

We further consider the case of upper half-plane and therefore the SIE assumes the following form

$$
\left(e^{-i \beta}+e^{i \beta}\right)\left(\kappa g^{\prime}-\bar{g}^{\prime}\right)+\kappa\left(e^{-i \beta}-e^{i \beta}-\frac{\beta_{z}^{\prime}}{\beta_{\zeta}^{\prime}} e^{-i \beta}\right) \mathbf{S}\left(g^{\prime}\right)-\left(e^{-i \beta}+e^{i \beta}-\kappa \frac{\beta_{z}^{\prime}}{\beta_{\zeta}^{\prime}} e^{i \beta}\right) \overline{\mathbf{S}\left(g^{\prime}\right)}=0, \quad \zeta \in \Gamma .
$$

This equation is the homogeneous dominant SIE, a part of the full SIE of the form investigated by Gakhov [7]. Namely

$$
a g+b \bar{g}+c \mathbf{S} g+d \overline{\mathbf{S} g}+\mathbf{K} g+\overline{\mathbf{L} g}=f .
$$

Here $f=0$ and the coefficients $a, b, c, d$ are known continuous functions of the complex contour variable specified as follows

$\left.a=\kappa\left(e^{-i \beta}+e^{i \beta}\right), b=-e^{-i \beta}-e^{i \beta}\right), c=\kappa\left(e^{-i \beta}-e^{i \beta}-\frac{\beta_{z}^{\prime}}{\beta_{\zeta}^{\prime}} e^{-i \beta}\right), d=-e^{-i \beta}-e^{i \beta}+\kappa \frac{\beta_{z}^{\prime}}{\beta_{\zeta}^{\prime}} e^{i \beta}$.

The regular operators $\mathrm{Kg}$ and $\mathrm{Lg}$ can be found from the full SIE (26), in the case of halfplane they vanished. It has been shown [7] that the solvability of SIE (33) is determined by the index calculated through the increment of the argument of the complex valued function $G$ after the complete traverse of $\Gamma$ in the counter-clockwise direction

$$
\begin{aligned}
& 2 \mathrm{~K} \equiv \operatorname{Index}(G)=\left.\frac{1}{2 \pi i}[\ln (G)]\right|_{\Gamma}=\left.\frac{1}{2 \pi}[\arg (G)]\right|_{\Gamma} \\
& G=\frac{A-i B}{A+i B}, \quad A-i B=(\bar{a}+\bar{c})(a-c)+(b+d)(\bar{d}-\bar{b}) .
\end{aligned}
$$

If $2 \mathrm{~K}>0$ (the coefficient 2 is introduced for convenience), then the solution of the homogeneous equation SIE $(f=0)$ depends on $2 \mathrm{~K}$ complex constants or $4 \mathrm{~K}$ real constants, otherwise the SIE does not have solutions, see Gakhov [7].

\subsection{Special case of boundary conditions}

Let us consider the case $\beta_{n}^{\prime}(\zeta)=0$, which can be important for some applications in geomechanics. In this case the coefficients $a, b, c, d$ become

$$
a=\kappa\left(e^{-i \beta}+e^{i \beta}\right), b=-e^{-i \beta}-e^{i \beta}, c=-\kappa e^{i \beta}, d=(\kappa-1) e^{i \beta}-e^{-i \beta} .
$$

Therefore

$$
A-i B=4 \kappa \cos \beta\left(\kappa e^{i \beta}-e^{-i \beta}\right)
$$

and consequently

$$
G=\frac{A-i B}{A+i B}=e^{2 i \beta} \frac{\kappa e^{i \beta}-e^{-2 i \beta}}{\kappa-e^{2 i \beta}} .
$$

Since $\kappa>1$ the fraction in (38) does not affect the index as its real part is always positive, therefore, the index is found as follows 


$$
2 \mathrm{~K} \equiv \operatorname{Index}(G)=\frac{1}{2 \pi i}\left[\left.\ln (\exp (2 i \beta)]\right|_{\Gamma}=\left.\frac{\beta}{\pi}\right|_{\Gamma} .\right.
$$

This result indicates that the index depends on the displacement orientations only, i.e. on the angle $\beta$, whose increment (after the complete traverse of $\Gamma$ in the positive direction) should be positive in order to provide a solution of the considered BVP. If $2 \mathrm{~K} \leq 0$, no bounded (non-trivial) solutions exist. For $2 \mathrm{~K}>0$ the solution of (32) for the special case of the coefficients (36) includes an arbitrary polynomial of $2 \mathrm{~K}-1$ degree, which means that the solution is controlled by up to $4 \mathrm{~K}$ real parameters.

\subsection{Numerical approach}

Direct application of any boundary element technique to the homogeneous dominant SIE of the form (32) would produce only a trivial solution due to $f=0$. Therefore we should transform this SIE such that its right-hand side becomes non-homogeneous and none of the homogeneous solutions is lost.

It has already been shown in [5] that application of the following integral operator

$$
\boldsymbol{\Theta}(.)=\bar{a} \mathbf{I}(.)-b \overline{\mathbf{I}(.)}-\bar{c} \mathbf{S}(.)-d \overline{\mathbf{S}(.)}
$$

(I is the identical operator, i.e. $\mathbf{I} f=f$ ) reduces the dominant eqn (32) (as well as the full SIE (26)) to the form

$$
\begin{gathered}
A g^{\prime}-i B \mathbf{S} g^{\prime}+\operatorname{Reg}\left(g^{\prime}, \bar{g}^{\prime}\right)=0, \\
A=a \bar{a}-b \bar{b}-c \bar{c}+d \bar{d}, \quad i B=\bar{a} c-a \bar{c}+\bar{b} d-b \bar{d}, \quad \operatorname{Im}(A)=\operatorname{Im}(B)=0 .
\end{gathered}
$$

We further seek the solution of (41) as the sum of two functions

$$
g^{\prime}=g_{0}^{\prime}+g_{1}^{\prime} \text {. }
$$

Here $g_{0}^{\prime}$ is the general solution of the homogeneous dominant equation

$$
A g_{0}-i B \mathbf{S} g_{0}=0
$$

and $g_{1}^{\prime}$ is any particular solution of the non-homogeneous SIE

$$
A g_{1}^{\prime}-i B \mathbf{S} g_{1}^{\prime}+\operatorname{Reg}\left(g_{1}^{\prime}, \bar{g}_{1}^{\prime}\right)=-\operatorname{Reg}\left(g_{0}^{\prime}, \bar{g}_{0}^{\prime}\right)
$$

where the right hand side is expressed through the analytical solution of SIE (43); it includes all linearly independent solutions of (43) and hence (44) is not homogeneous anymore. Therefore, any proper boundary element method can be applied to find the numerical solution of the non-homogeneous SIE (44). Finally the sum in (42) determines the solution of the homogeneous dominant SIE, where the analytical part $g_{0}^{\prime}$ is found in Gakhov [7]. Further on the solution for the contour derivative of the displacement vector is found from (7).

It should be noted that this algorithm without any modifications can be applied for solving the full SIE as well.

\section{CONCLUSIONS}

In this paper we investigated the solvability of an incorrectly posed BVP of the Cauchy type for elastic half-plane formulated in terms of the orientations of the displacements vector and its normal derivative on the boundary. This study complements the method of 
investigation proposed in [1] by reducing the full SIE of the form that contains both unknown function $g(\zeta)$ and its derivative to the SIE of the form as in (26) and then to the SIE of the form as in (33) that has been investigated earlier. A numerical approach capable of dealing with both homogeneous and non-homogeneous SIEs has been proposed.

\section{ACKNOWLEDGEMENT}

The author is thankful to the Russian Foundation for Basic Research that supported this work by the Grant 16-01-00260.

\section{REFERENCES}

[1] Galybin, A.N. \& Mukhamediev, Sh.A., Plane elastic boundary value problem posed on orientation of principal stresses. Journal of the Mechanics and Physics of Solids, 47(11), pp. 2381-2409, 1999. DOI: 10.1016/s0022-5096(99)00032-0.

[2] Galybin, A.N. \& Mukhamediev, Sh.A., Integral equations for elastic problems posed in principal directions: application for adjacent domains. WIT Transactions on Modelling and Simulation, vol. 42, WIT Press: Southampton and Boston, pp. 51-60, 2006.

[3] Galybin, A.N., Boundary value problems of plane elasticity involving orientations of displacements and tractions. Journal of Elasticity, 102(1), pp. 15-30, 2011.

DOI: 10.1007/s10659-010-9259-4.

[4] Galybin, A.N., Boundary integral equations for plane elastic problems posed in terms of stress orientations. Proceedings of the 8th UK Conference on Boundary Integral Methods, University of Leeds, UK, 2011.

[5] Galybin, A.N., On solvability of the BVP formulated in terms of displacement orientations on the interface between dissimilar elastic materials. International Journal of Computational Methods and Experimental Measurements, 5(3), pp. 369376, 2017. DOI: 10.2495/cmem-v5-n3-369-376.

[6] Galybin, A.N., Boundary value problems for elastic half-planes posed in terms of stress and displacement orientations. International Journal of Computational Methods and Experimental Measurements, 6(6), pp. 1173-1181, 2018.

DOI: 10.2495/cmem-v6-n6-1173-1181.

[7] Gakhov, F.D., Boundary Value Problems. Nauka (3rd Russian edition, see also the translation of the first edition by Pergamon Press, 1966: Moscow), 1977.

[8] Muskhelishvili, N.I., Some Basic Problems of the Mathematical Theory of Elasticity, The Netherlands, 1963. 